\title{
Electric fields generated by synchronized oscillations of microtubules, centrosomes and chromosomes regulate the dynamics of mitosis and meiosis
}

\author{
Yue Zhao ${ }^{*}$ and Qimin Zhan
}

\author{
* Correspondence: alexanderyz@ \\ gmail.com \\ State key laboratory of molecular \\ oncology, Cancer Institute \& \\ Hospital of Chinese Academy of \\ Medical Sciences, Peking Union \\ Medical College, Room 6107, No.17 \\ Pan Jia Yuan Nan Li, Chao Yang \\ District, Bei Jing 100021, China
}

\begin{abstract}
Super-macromolecular complexes play many important roles in eukaryotic cells. Classical structural biological studies focus on their complicated molecular structures, physical interactions and biochemical modifications. Recent advances concerning intracellular electric fields generated by cell organelles and super-macromolecular complexes shed new light on the mechanisms that govern the dynamics of mitosis and meiosis. In this review we synthesize this knowledge to provide an integrated theoretical model of these cellular events. We suggest that the electric fields generated by synchronized oscillation of microtubules, centrosomes, and chromatin fibers facilitate several events during mitosis and meiosis, including centrosome trafficking, chromosome congression in mitosis and synapsis between homologous chromosomes in meiosis. These intracellular electric fields are generated under energy excitation through the synchronized electric oscillations of the dipolar structures of microtubules, centrosomes and chromosomes, three of the super-macromolecular complexes within an animal cell.
\end{abstract}

Keywords: Polar wind, Chromosome, Microtubule, Centrosome, Spindle body

\section{Background}

The choreography of microtubules, centrosomes and chromosomes during mitosis and meiosis is beautifully designed by nature. Finely regulated and synchronized movements of these super-macromolecular complexes against the entropic forces within a dividing cell ensure the fidelity of the genetic material in both daughter cells. Currently, several models exist for the mechanisms of chromosome congression and spindle body assembly during $M$ phase such as the search and capture model, kinetochoremediated k-fibre formation, kinetochore motors contributing to congression, and the polar wind model. The mechanisms evoked by these models probably overlap, so there is redundancy among them, since mutations in the genes involved have only mild effects on chromosome congression during mitosis [1]. Many open questions remain within these models. In the polar-wind model, an unknown force (also known as the ejection force) generated by the spindle poles is considered to push the chromosomes to the spindle equator. Laser microsurgery experiments show that chromosome

(c) 2012 Zhao and Zhan; licensee BioMed Central Ltd. This is an Open Access article distributed under the terms of the Creative Commons Attribution License (http://creativecommons.org/licenses/by/2.0), which permits unrestricted use, distribution, and reproduction in any medium, provided the original work is properly cited. 
fragments without kinetochores are invariably expelled from the spindle, and chromosomes without kinetochores can still move from the vicinity of the spindle pole to the spindle equator [1-3]. The ejection force of the spindle body is dependent on the polymerization of spindle body microtubules, as depolymerization of astral microtubules by nocodazole or colcemid prevents the expulsion of severed chromosome arms from the spindle, whereas stabilization of microtubules by taxol drives chromosomes to the periphery of the astral array [4]. In addition, the driving force responsible for the pole-ward flux of spindle microtubules during metaphase remains uncharacterized [5].

Cellular electric fields have been studied in various cell types, and several studies have reported the existence of dielectrophoretic forces around cells [6-8]; electromagnetic interactions between cells have also been studied [9-11]. Cifra et al. proposed that microtubules, which comprise heterodimers polymerized into a helical structure, can generate an electric field under intracellular energy excitation [12-15]. Inhibition of microtubule polymerization by an external electromagnetic field has been reported by Kirson et al. $[16,17]$. Pokorny' et al. detected four peaks of electric field activity around yeast cells during $\mathrm{M}$ phase, which correlated with spindle body assembly, kinetochore microtubule capture, and mitotic spindle elongation during anaphase A and B, visualized by fluorescence microscopy [18]. Comparing synchronized and unsynchronized tubulin mutants of yeast cells, Pokorny' et al. verified that synchronized yeast cells show more electric activity during $M$ phase than non-synchronized yeasts [19]. Direct measurements of electric resonant oscillations in microtubules have been presented at conferences by A. Bandyopadhyay. The technical aspects of direct detection of electric fields within a living cell have been discussed in a recent review [20]. Resonance absorption of external electromagnetic fields by cancers has been reported by Vedruccio et al. [21], and Zimmerman et al. reported that cancer cell proliferation is inhibited by specific modulation frequencies [22].

Coherent oscillations in microtubules can be explained by Fröhlich's theory, which describes a system of oscillators with energy supply, linear and nonlinear coupling with a heat bath. If a sufficient energy supply is provided to this system, condensation of energy occurs in the lowest mode leading to its coherent excitation [23,24]. Electrostatic but not electrodynamic interactions are screened over long distances (Debye Screening). Given an intracellular salt concentration of $\sim 150 \mathrm{mM}$, the effectiveness of electrostatic interaction is shortened to the nanometer range (the Debye length is $\sim 0.7-0.8 \mathrm{~nm}$ ). However, resonant electrodynamic interactions, such as the electromagnetic interactions generated by electric oscillations within the cell, may play a role in the long-distance recruitment of biomolecules. Following Fröhlich, Preto et al. suggested that long-range electrodynamic interactions can be triggered only under resonance conditions, and such interactions are effective when one normal mode is statistically privileged, typically out of thermal equilibrium, which could be the case in the intracellular context $[25,26]$.

In this article, we integrate research from several disciplines to provide an 'electric' view of the dynamics of these super-macromolecular complexes in mitosis, meiosis and other relevant cellular events. From our theoretical point of view, many of the unidentified forces regulating major cellular dynamic events during mitosis are probably electric forces generated by the synchronized oscillation of the electric dipoles within these super-macro organelle structures. Chronic exposure to extremely low frequency electric fields could affect several key steps of mitosis and neuronal cell physiology, resulting in an increased risk for cancer. 


\section{The electrical properties of microtubules and centrosomes}

The electric field of the microtubule is generated by the synchronized oscillation of $\alpha$ and $\beta$ tubulins. These tubulins form electric dipoles during microtubule polymerization; under intracellular energy excitation, synchronized oscillation of $\alpha$ and $\beta$ tubulin subunits generates a longitudinal electric field around the microtubule [12-15] (Figure 1). Cifra et al. suggested that the source of the energy excitation could be hydrolysis of guanosine triphosphate (GTP) during the process of dynamic instability of microtubules, and also energy transferred from the movement of motor proteins or released from mitochondria as "wasted" energy from the citric acid cycle. We propose that the overall entropic environment within a living cell could be the source of energy for electric oscillation of microtubules. Cancer cells have different entropic states from normal cells as a result of the Warburg effect, which can cause mitochondrial malfunctions and further lead to alteration of cytoskeleton-based cellular elastoelectrical oscillations [27]. The microtubule networks of cancer cells generate an electromagnetic field with different frequencies. Thus, specific electromagnetic frequencies have been used to diagnose specific cancers [21,28], and tumor-specific modulating electromagnetic fields have been used to treat patients with advanced cancer with positive results [22,29].

The centriole of the centrosome is composed of $\alpha, \beta$ and $\gamma$ tubulins organized differently from the subunits of microtubules; each centrosome comprises two centrioles, which are composed of nine triplets of microtubules. The two centrioles are arranged perpendicularly and surrounded by an amorphous mass of dense material (the pericentriolar material) [30]. As in microtubules, an electric field would be generated by synchronized oscillation between the $\alpha$ and $\beta$ tubulins within the microtubule triplet of the centrioles (Figure 2).

\section{Electric fields in centrosome separation and bipolar spindle body assembly}

Mechanisms of centrosome separation and bipolar spindle body assembly have been discussed in a recent review [31]. The process is still incompletely understood. Plus end-directed motor proteins such as kinesin 5 and minus end-directed motor proteins such as dynein are known to play dominant roles in centrosome separation and spindle assembly. However, centrosomal microtubules and microtubules of the nuclear envelope (NE) and cellular cortex need to move into close proximity for motor proteins to attach to both so they can generate the pulling forces. The current models assume a

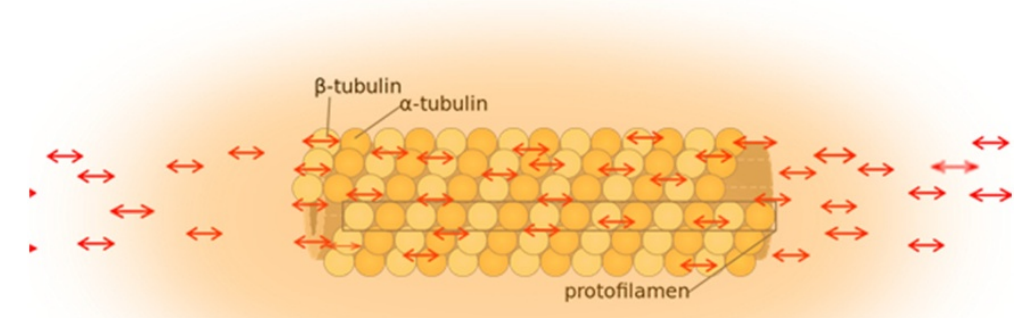

Figure 1 The red arrow illustrates the electric field of the microtubule under intracellular energy excitation. 


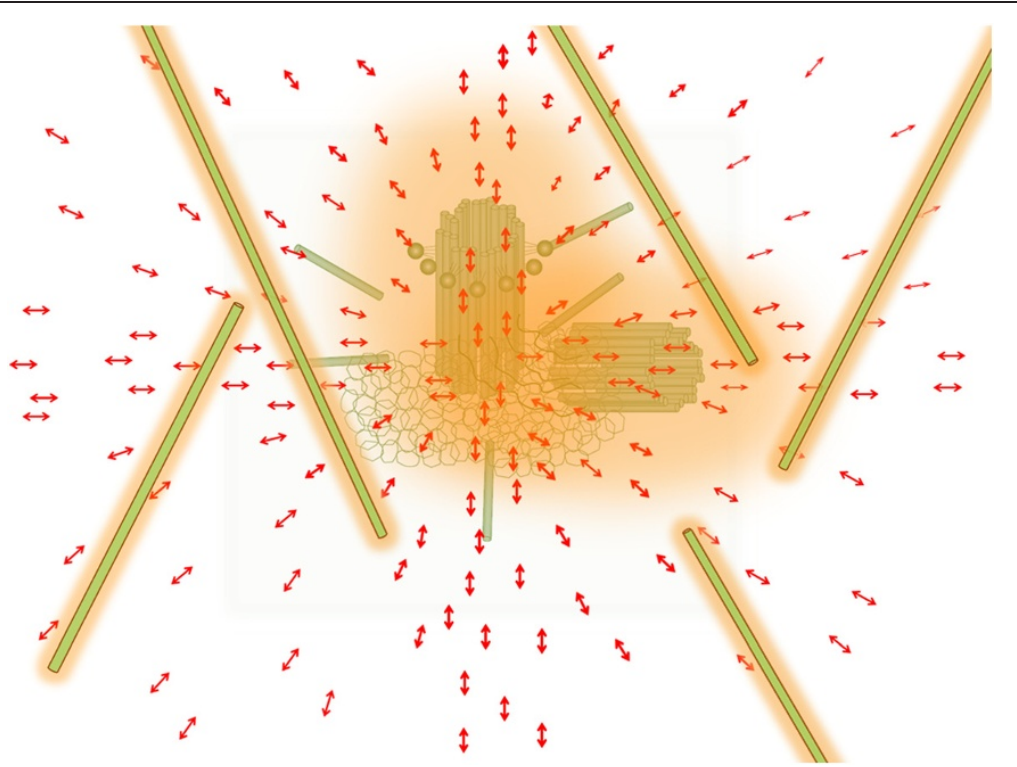

Figure $\mathbf{2}$ The red arrows illustrate the electric field of a centrosome under intracellular energy excitation.

randomized mode of microtubule interaction, which is quite inefficient. For example, at a certain point a centrosome would have to stop moving until certain microtubules had grown sufficiently for appropriate bridging by motor proteins, particularly during prophase, when the centrosomes do not have many associated microtubules. When the electric fields of microtubules and centrosomes are considered, these structures are mutually attractive. Thus, centrosome movement along the microtubule networks of the cellular cortex and NE is more efficient. We can also envision a more autonomous mode of microtubule lattice formation within the cellular cortex and NE.

\section{Electrical properties of duplicated chromosomes}

Andrews et al. have studied the effects of high frequency (range 2 to $50 \mathrm{MHz}$ ) electric fields on mammalian (human and Chinese hamster) chromosomes in vitro. They showed that such chromosomes can be oriented, aligned and translated by an oscillating electrical force. They also observed that above certain threshold field strengths the chromosomes orient themselves with their long axes along the field direction. The dependence of this threshold on frequency was measured and was found to be much larger at low than at high frequencies [32]. Using electric dichroism experiments, Crothers reported permanent dipole moments in dinucleosomes linked by 140 and 175 base pairs of DNA [33]. Jian Sun et al. suggested an electrostatic mechanism of nucleosomal array folding, revealed by computer simulation, which explains the saltdependent chromatin fiber conformations [34]. Schalch et al. reported that the X-ray structure of an oligonucleosome revealed that linker DNA elements zigzag back and forth between two stacks of nucleosome cores, forming a truncated two-start helix, and do not follow a path compatible with a one-start solenoidal helix [35]. Grigoryev et al. reported evidence for heteromorphic chromatin fibers, showing that the 2-start zigzag topology and the type of linker DNA bending that defines solenoid models may be 
simultaneously present in a structurally heteromorphic chromatin fiber with a uniform $30 \mathrm{~nm}$ diameter [36].

However, the physical mechanisms that regulate higher order packaging of $\mathrm{M}$ phase chromosomes are still not well characterized. Here we present a hypothesis of chromosome compaction. We apply a pulse-coupled oscillation clustering model to the dynamic events of chromosome packaging and inter-/intra-chromosomal organization. During chromosomal packaging, differentially compacted regions form partially synchronized electric oscillators interacting with an elastic electromagnetic field. According to the physical pulse-coupled oscillator model, unsynchronized pulse-coupled oscillators with proximal natural frequencies form synchronized oscillation clusters at a given coupling strength. As the coupling strength increases, these synchronized oscillation clusters merge with each other [37-40].

During $\mathrm{M}$ phase chromosome compaction, the $30 \mathrm{~nm}$ chromatin fiber is initially formed by the electrostatic forces between neighboring nucleosomes. Under intracellular stochastic energy excitation, electric dipolar oscillation would be generated between neighboring nucleosomes. After oscillation synchronization and coupling, regulated electric oscillation is generated along the $30 \mathrm{~nm}$ chromatin fiber, and the oscillation coupling process further compacts that fiber [12-14,41]. This facilitates further packing into the $300 \mathrm{~nm}$ fiber; the electric field bends according to the physical curvature of the compacting $30 \mathrm{~nm}$ fiber, generating an oscillating electromagnetic field that goes through the $300 \mathrm{~nm}$ chromatin fiber. After the second round of oscillation synchronization and coupling, the $300 \mathrm{~nm}$ fiber becomes compacted and coiled into the $250 \mathrm{~nm}$ chromatin fiber, along which the third order of electromagnetic field is generated; this round of oscillation coupling and clustering facilitates the packing of the $250 \mathrm{~nm}$ chromatin fiber into the $700 \mathrm{~nm}$ chromosome arms. The coiling electromagnetic field of the $250 \mathrm{~nm}$ chromatin fiber generates the electromagnetic field of a chromosome arm $[42,43]$ (Figure 3). We speculate that the source of the dipolar electric oscillation between neighboring nucleosomes is the variety of intracellular entropic forces, and the direction of oscillation primarily depends on the zigzag arrangement of neighboring nucleosomes along the $30 \mathrm{~nm}$ chromatin fiber. Each of the $\mathrm{M}$ phase chromosomal arms can be viewed as a partially synchronized oscillation cluster. Under intracellular energy excitation, partially synchronized electric fields can be generated for each chromosomal arm, and these orient the dynamics of the chromosome when they interact with the electric field generated by the spindle microtubules.

Theoretically, the oscillation clustering model explains the closely juxtaposed configuration of duplicated chromosomes during $M$ phase, which is counter-intuitive from the perspective of electrostatic repulsion between duplicated chromosome arms. As the homologous chromosomal regions develop synchronized oscillation clusters with identical natural frequencies, they tend to cluster together. The same scenario could apply to synapsis during meiosis; the electric oscillations of homologous chromosomes couple with each other, preventing synapsis between non-homologous chromosomes.

\section{Electric interactions during mitosis and meiosis}

The intracellular electric fields described in the foregoing sections could facilitate several cellular events during mitosis and meiosis. First, the metaphase bipolar spindle microtubules 


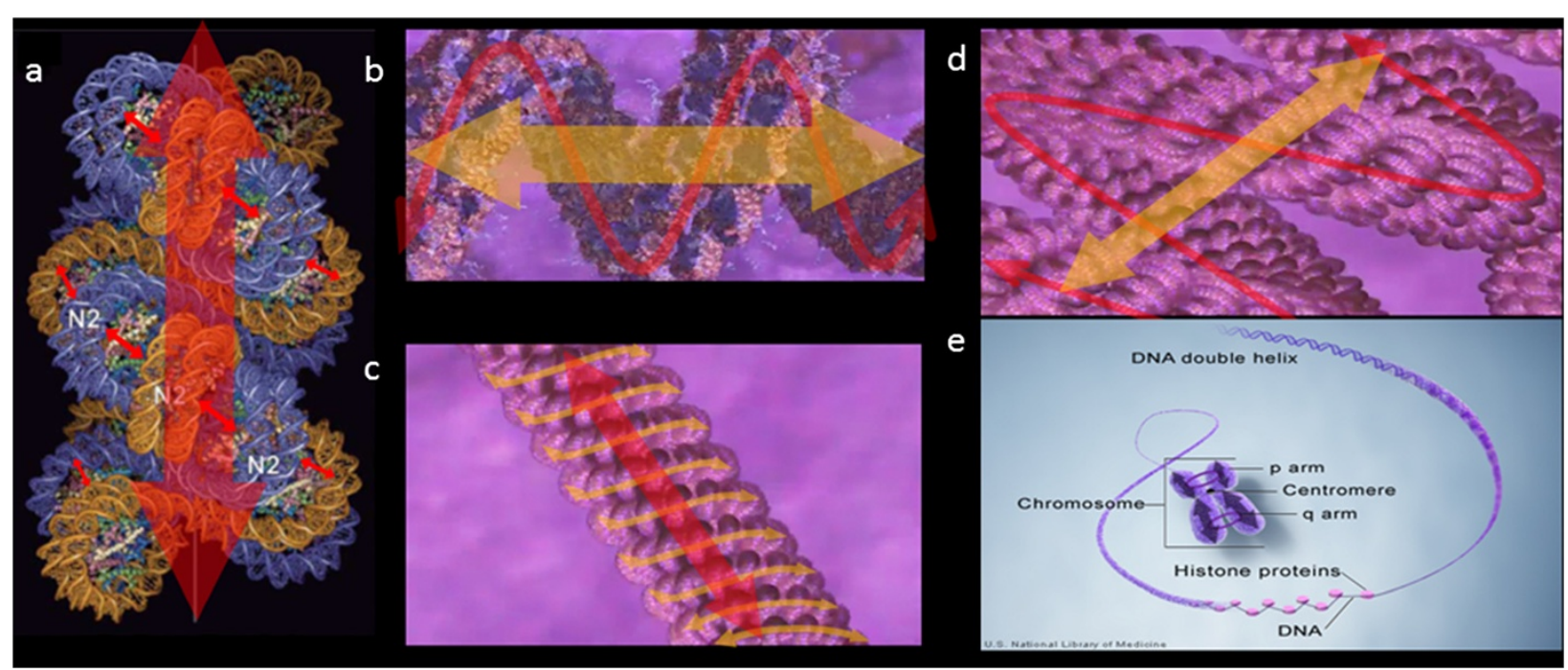

Figure 3 (a) The small red arrows indicate the electric oscillations generated between the neighboring histone octamers by excitation of entropic energy within the cell nucleus. The big red arrow represents the electric field generated by the electric oscillation along the $30 \mathrm{~nm}$ chromatin fiber. (b-d) Schematic illustration of several orders of oscillation coupling and clustering of EMFs in chromatin fibers, which facilitate the multi-step event of M phase chromosome packaging. The red and orange arrows indicate the multiple orders of EMFs generated during

chromosome packaging. (e) The purple arrows indicate the EMFs of compacted M phase chromosome arms; the purple cycles indicate coupling of EMFs. The duplicated chromosome arms hold a juxtaposed position. 
are formed through microtubule nucleation from the $\gamma$ tubulin ring complexes ( $\gamma$-TuRCs) at the centrosomes and retrograde delivery of peripheral microtubules by motor proteins [31,44-46]. The electric fields of microtubules and centrosomes could facilitate spindle microtubule assembly through electric interactions. Secondly, the unidentified polar-wind or ejection force of the spindle body is likely to be generated by interactions between the electric fields of the spindle body and chromosomes [1-3]. In this case, the bipolar spindle body and chromosomes can be viewed as two oscillating clusters with different average oscillating frequencies. Given the oscillation clustering model, a partially entrained system of oscillators with similar frequencies preferentially cluster with each other at a given coupling strength [37-40]. So the clustering of electric fields of spindle microtubules would result in the repulsion of the electric field of the chromosomes, which pushes the chromosomes from the proximal regions of centrosomes out of the spindle body. Congression at the central plate regions of a dividing cell can also be viewed as oscillation clustering of the electric fields of chromosomes. The processes may also be viewed as a dynamic electric phase, which indicates that the intensity of the electric field changes in different subcellular regions during metaphase. Cell organelles with different electric field intensities would automatically locate themselves according to the electric phase. In addition, the pole-ward flux of spindle microtubules during metaphase could be driven by the electric locking of those microtubules within the spindle body, which means that the synchronized electric fields of the spindle body would hold the physical position of a spindle microtubule growing at the plus end and depolymerizing at the minus end at the same time within a dividing cell [5] (Figure 4).

According to the physical organization of the duplicated chromosome arms, the condensed electric chromosomal fields around the centromeric regions could attract microtubule fragments to the sister kinetochores through electric interaction, which is consistent with observations of kinetochore movement along uncaptured microtubules, forming K-fibers (kinetochore associated microtubules) [47]. Thus, electric interactions between chromosomes and microtubules may also facilitate K-fiber capture by

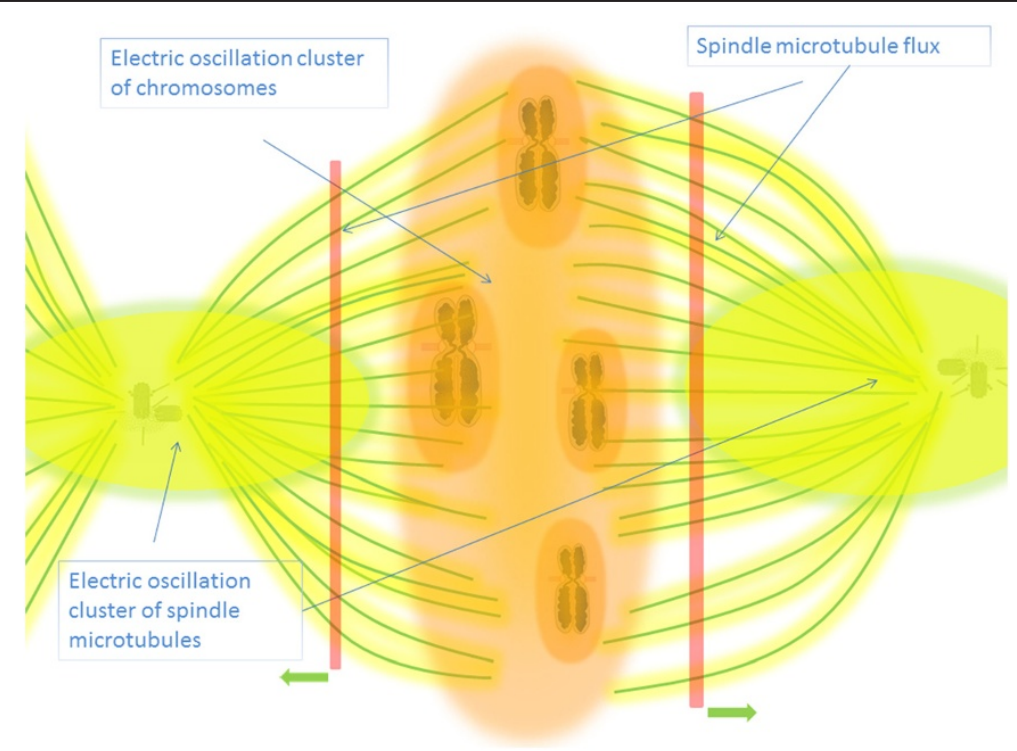

Figure 4 Schematic illustration of the electric interactions between spindle body microtubules and chromosomes facilitating congression through oscillation clustering, and spindle body pole-ward flux during mitosis; the green arrows indicate the direction of the pole-ward flux. 
kinetochores. The chromosome oscillation observed during congression could be explained as the turbulence of chromosome arms passing through the chaotic electric landscape of two astral microtubule networks.

In meiosis, the kinetochores are positioned at one side of the duplicated chromosome dimers, so the two sister chromosomes do not separate. The electric oscillation clustering between homologous chromosomal regions results in synapsis and recombination between homologous chromosomes; the electric fields generated by two duplicated homologous chromosomes can be viewed as two identical partially-entrained electric oscillation clusters, constituted by sub-chromosomal clusters throughout the chromosome arms. Such clusters in homologous chromosomes share identical electric frequencies, so the close juxtaposition between homologous chromosomes at synapsis is achieved through electric clustering and coupling among them. Synapsis does not occur during mitosis probably because the chromosome configuration caused by the opposing outward-pulling forces of the kinetochores at the opposite sides of duplicated centromere disfavors inter-chromosomal electric attraction. In addition, this event may be regulated by synaptonemal complex proteins [48].

Magidson et al. reported that chromosomes adopt a toroidal/ring shape organization of after NE breakdown, which facilitates spindle assembly during $M$ phase [47]. Their observation matches the electric model at several points: the ring shape organization could be generated by the electric interaction between $M$ phase chromosomes and the spindle body, and the interplay between the electric fields of the chromosome ring and spindle body microtubules promotes the capture of microtubules by kinetochores.

\section{Discussion}

Numerous reports indicate that extremely low frequency electric fields can increase the risks of certain types of cancer [49]. Micronuclei (MN) in buccal mucosal cells, comprising acentric fragments or complete chromosomes that fail to attach to the mitotic spindle during cytokinesis, are increased in people chronically exposed to extremely low frequency electric fields [50]. Research by Hardell et al. indicates increased brain tumor risks with latency time and cumulative mobile or cordless phone use [51]. Volkow et al. reported that 50-minute cell phone exposure was associated with increased brain glucose metabolism in the region closest to the antenna [52]. However, the exact cellular biophysical pathways that relay very low frequency electric radiations to genetic alterations that lead to cancer are not well characterized. From our theoretical point of view, chronic exposure to extremely low frequency electric fields would intervene in several key steps of mitosis and neuronal cell physiology, potentially resulting in an increased risk for cancer.

To characterize these intracellular electric fields and study their cellular functions further, biophysicists should develop more detailed mathematical and physical models for chromosome electric fields and their role in $\mathrm{M}$ phase chromosome compaction, to allow these fields to be described and calculated more precisely and to predict the dynamics of related cellular events. The dynamic of changes of the electric fields in a living cell during mitosis and other cellular processes could be visualized using live cell imaging technologies such as nano-sized voltmeters [53]. It would be particularly interesting to observe microtubule self-organization under energy excitation in vitro, which would allow us to observe the dynamics of microtubule movements directly through electric interactions. These insights will help us to understand the molecular mechanisms 
of signal pathways better and to elucidate cellular super-macromolecular behavior, cell organelle organization and functions, intra- and inter-cellular communications, tissue morphogenesis, embryo development, neurobiology, and oncogenesis, and finally to advance our knowledge about life to a new level.

Competing interests

The authors declare they have no competing interests.

\section{Authors' contributions}

Yue Zhao conceived the general concepts and wrote the article; Qimin Zhan shared insights and gave advice. Both authors read and approved the final version of the article.

\section{Acknowledgments}

We thank Dr. Michal Cifra of Institute of Photonics and Electronics, Academy of Sciences of the Czech Republic and Dr. Yujie Sun of Biodynamic Optical Imaging Center of Peking University for their helpful discussion and insights about electric fields in live intracellular organelles. We thank Dr. Lennart Hardell of University Hospital, Sweden for providing us some of the latest epidemiological data linked with EMF and cancer. We also thank Editor-in-Chief of Theoretical Biology and Medical Modelling Dr. Paul Agutter for language editing of the article.

Received: 1 May 2012 Accepted: 26 June 2012

Published: 2 July 2012

\section{References}

1. Walczak CE, Cai S, Khodjakov A: Mechanisms of chromosome behavior during mitosis. Nat Rev Mol Cell Bio 2009, 11:91-102

2. Rieder CL, Davison EA, Jensen LCW, Cassimeris L, Salmon ED: Oscillatory movements of monooriented chromosomes and their position relative to the spindle pole result from the ejection properties of the aster and the half-spindle. J Cell Biol 1986, 103:581-591.

3. Khodjakov A, Rieder CL: Kinetochores moving away from their associated pole do not exert a significant pushing force on the chromosome. J Cell Biol 1996, 135:315-327.

4. Ault JG, DeMarco AJ, Salmon ED, Rieder CL: Studies on the ejection properties of asters: astral microtubule turnover influences the oscillatory behavior and positioning of mono-oriented chromosomes. J Cell Sci 1991, 99:701-710.

5. Kwok BH, Kapoor TM: Microtubule flux: drivers wanted. Curr Opin Cell Biol 2007, 19:36-42.

6. Pohl J, Christophers E: Photo inactivation and recovery in skin fibroblasts after formation of mono- and bifunctional adducts by furocoumarins-plus-UVA. J Invest Dermatol 1980, 75(4):306-310.

7. Hölzel R, Lamprecht I: Electromagnetic fields around biological cells. Neural Net World 1994, 4(3):327.

8. Hölzel R: Electric Activity of Non-Excitable Biological Cells at Radio Frequencies. Electro- and Magnetobiol 2001, 20:1.

9. Albrecht-Buehler G: Surface extensions of 3 T3 cells towards distant infrared sources. J Cell Biol 1991, 114:493-502.

10. Albrecht-Buehler G: A rudimentary form of cellular vision. Proc Natl Acad Sci USA 1992, 89:8288-8292.

11. Albrecht-Buehler G: A Long-Range Attraction Between Aggregating 3T3 Cells Mediated By Near-Infrared Light Scattering. Proc Natl Acad Sci USA 2005, 102(14):5050-5055.

12. Havelka D, Cifra M: Calculation of the electromagnetic field around microtubule. Acta Polytechnica Czech Technical University in Prague. CTU Publishing House 2009, 49(2-3):58-63. ISSN 1210-2709.

13. Cifra M, Havelka D, Deriu MA: Electric Field Generated by Longitudinal Axial Microtubule Vibration Modes with High Spatial Resolution Microtubule Model. Electrodynamic Activity of Living Cells. J Physics: Conference Series 2011, 329:012013.

14. Cifra M, Pokorný J, Havelka D, Kucera O: Electric field generated by axial longitudinal vibration modes of microtubule. Biosystems 2010, 100(2):122-131.

15. Havelka D, Cifra M, Kučera O, Pokorný J, Vrba J: High-frequency electric field and radiation characteristics of cellular microtubule network. J Theor Biol 2011, 286(1):31-40.

16. Kirson ED, Gurvich Z, Schneiderman R, Dekel E, Itzhaki A, Wasserman Y, Schatzberger R, Palti Y: Disruption of Cancer Cell Replication by Alternating Electric Fields. Cancer Res 2004, 64:3288-3295.

17. Kirson ED, Dbalý V, Tovarys F, Vymazal J, Soustiel JF, Itzhaki A, Mordechovich D, Steinberg-Shapira S, Gurvich Z, Schneiderman R, Wasserman Y, Salzberg M, Ryffel B, Goldsher D, Dekel E, Palti Y: Alternating electric fields arrest cell proliferation in animal tumor models and human brain tumors. Proc Natl Acad Sci USA 2007, 104 (24):10152-10157.

18. Pokorny J, Hasek J, Jelınek F, Saroch J, Palan B: Electric activity of yeast cells in the M phase. Electro Magnetobiol 2001, 20:371-396.

19. Jelínek F, Cifra M, Pokorny J, Vanis J, Simsa J, Hasek J, Frydlová I: Measurement of Electrical Oscillations and Mechanical Vibrations of Yeast Cells Membrane Around 1kHz. Electric Biology and Medicine 2009, 28(2):223-232.

20. Kučera O, Cifra M, Pokorný J: Technical aspects of measurement of cellular electric activity. Eur Biophys J 2010, 39(10):1465-1470.

21. Vedruccio C, Meessen A: EM Cancer Detection by Means of Non Linear Resonance Interaction. Pisa, Italy: Proceedings of PIERS 2004, Progress in Electromagnetics Research Simposium; 2004:909-912.

22. Zimmerman JW, Pennison MJ, Brezovich I, Yi N, Yang CT, Ramaker R, Absher D, Myers RM, Kuster N, Costa FP, Barbault A, Pasche B: Cancer cell proliferation is inhibited by specific modulation frequencies. Br J Cancer 2012, 106:307-313. doi:10.1038/bjc.2011.523.

23. Fröhlich H: Long-range coherence and energy storage in biological systems. International Journal of Quantum Chemistry 1968, 2:641-649. 
24. Fröhlich $\mathrm{H}$ : The biological effects of microwaves and related questions. Advances in Electronics and Electron Physics 1980, 53:85-152.

25. Preto J, Floriani E, Nardecchia I, Ferrier P, Pettini M: Experimental assessment of the contribution of electrodynamic interactions to long-distance recruitment of biomolecular partners, theoretical basis. Phys Rev E Stat Nonlin Soft Matter Phys 2012, 85(4-1):041904. PMID 22680495.

26. Preto J, Pettini M: Long-range resonant interactions in biological systems. arXiv, 120:5187v1.

27. Cairns RA, Harris IS, Mak TW: Regulation of cancer cell metabolism. Nat Rev Cancer 2011, 11(2):85-95.

28. Pokorný J, Vedruccio C, Cifra M, Kučera O: Cancer physics: diagnostics based on damped cellular elastoelectrical vibrations in microtubules. Eur Biophys J 2011, 40(6):747-759.

29. Barbault A, Costa FP, Bottger B, Munden RF, Bomholt F, Kuster N, Pasche B: Amplitude-modulated electromagnetic fields for the treatment of cancer: discovery of tumor-specific frequencies and assessment of a novel therapeutic approach. J Exp Clin Cancer Res 2009, 28(1):51.

30. Doxsey SJ: Re-evaluating centrosome function. Nature Rev Molec Biol 2001, 2:688-699.

31. Tanenbaum ME, Medema RH: Mechanisms of centrosome separation and bipolar spindle assembly. Dev Cell 2010, 14, 19(6):797-806.

32. Andrews MJ, McClure JA: Effects of high frequency electric fields on mammalian chromosomes in vitro. $J$ Biol Phys 1979, 6(1-2):69-86. doi:10.1007/BF02311220.

33. Crothers DM, Dattagupta N, Hogan M, Klevan L, Lee KS: Transient electric dichroism studies of nucleosomal particles. Biochemistry 1978, 17(21):4525-4533.

34. Sun J, Zhang Q, Schlick T: Electrostatic mechanism of nucleosomal array folding revealed by computer simulation. Proc Natl Acad Sci U S A 2005, 102(23):8180-8185.

35. Schalch T, Duda S, Sargent DF, Richmond TJ: X-ray structure of a tetranucleosome and its implications for the chromatin fibre. Nature 2005, 436:138-141.

36. Grigoryev SA, Arya G, Correll S, Woodcock CL, Schlick T: Evidence for heteromorphic chromatin fibers from analysis of nucleosome interactions. Proc Natl Acad Sci USA 2009, 106(32):13317-13322.

37. Strogatz SH, Stewart I: Coupled oscillators and biological synchronization. Sci Am 1993, 269(6):102-109.

38. Morelli LG, Cerdeira H, Zanette DH: Frequency clustering of coupled phase oscillators on small-world networks. Eur Phys J B 2005, 43:243-250

39. Smet FD, Aeyels $D$ : Clustering in a network of non-identical and mutually interacting agents. Proc $R \operatorname{Soc} A$ 2009, 465:745-768

40. Aeyels D, Smet FD: Emergence and evolution of multiple clusters of attracting agents. Physica D 2010 239:1026-1037.

41. Cifra M, Fields JZ, Farhadi A: Electromagnetic cellular interactions. Prog Biophys Mol Biol 2011, 105(3):223-246. doi:10.1016/j.pbiomolbio.2010.07.003.

42. Olins DE, Olins AL: Chromatin history: our view from the bridge. Nat Rev Mol Cell Biol 2003, 4:809-814 doi:10.1038/nrm1225.

43. Annunziato A: DNA packaging: Nucleosomes and chromatin. Nature Education 2008, 1(1).

44. Kollman JM, Merdes A, Mourey L, Agard DA: Microtubule nucleation by $\mathrm{y}^{-t u b u l i n}$ complexes. Nat Rev Mol Cell Biol 2011, 12:709-721.

45. Rusan NM, Tulu US, Fagerstrom C, Wadsworth P: Reorganization of the microtubule array in prophase/ prometaphase requires cytoplasmic dynein-dependent microtubule transport. J Cell Biol 2002, 158:997-1003

46. Tulu US, Rusan NM, Wadsworth P: Peripheral, non-centrosome-associated microtubules contribute to spindle formation in centrosome-containing cells. Curr Biol 2003, 13:1894-1899.

47. Magidson V, O'Connell CB, Lončarek J, Paul R, Mogilner A, Khodjakov A: The Spatial Arrangement of Chromosomes during Prometaphase Facilitates Spindle Assembly. Cell 2011, 146(4):555-567. PMID: 21854981

48. Petronczki M, Siomos MF, Nasmyth K: Un ménage à quatre: the molecular biology of chromosome segregation in meiosis. Cell 2003, 112(4):423-40.

49. Baan R, Grosse Y, Lauby-Secretan B, El Ghissassi F, Bouvard V, Benbrahim-Tallaa L, Guha N, Islami F, Galichet L, Straif K, WHO International Agency for Research on Cancer Monograph Working Group: Carcinogenicity of radiofrequency electric fields. Lancet Oncol 2011, 12(7):624-626.

50. Carbonari K, Gonçalves L, Roth D, Moreira P, Fernández R, Martino-Roth MG: Increased micronucleated cell frequency related to exposure to radiation emitted by computer cathode ray tube video display monitors. Genet Mol Biol 2005, 28(3):469-474.

51. Hardell L, Carlberg M, Mild KH: Pooled analysis of case-control studies on malignant brain tumours and the use of mobile and cordless phones including living and deceased subjects. Int J Oncol 2011, 38(5):1465-1474. doi:10.3892.

52. Volkow ND, Tomasi D, Wang GJ, Vaska P, Fowler JS, Telang F, Alexoff D, Logan J, Wong C: Effects of Cell Phone Radiofrequency Signal Exposure on Brain Glucose Metabolism. JAMA 2011, 305(8):808-813. doi:10.1001/ jama.2011.186.

53. Tyner KM, Kopelman R, Philbert MA: "Nanosized Voltmeter" Enables Cellular-Wide Electric Field Mapping. Biophys J 2007, 93:1163-1174.

doi:10.1186/1742-4682-9-26

Cite this article as: Zhao and Zhan: Electric fields generated by synchronized oscillations of microtubules, centrosomes and chromosomes regulate the dynamics of mitosis and meiosis. Theoretical Biology and Medical Modelling 2012 9:26. 